\title{
Insuficiência respiratória aguda como manifestação da síndrome de eosinofilia-mialgia associada à ingestão de L-triptofano*
}

\author{
Acute respiratory failure as a manifestation of eosinophilia-myalgia \\ syndrome associated with L-tryptophan intake \\ Tiago de Araujo Guerra Grangeia', Marcelo Schweller ${ }^{1}$, llma Aparecida Paschoal $^{2}$,
Lair Zambon ${ }^{3}$, Mônica Corso Pereira ${ }^{4}$
}

\section{Resumo}

A síndrome da eosinofilia-mialgia foi descrita em $1989 \mathrm{em}$ pacientes que apresentavam mialgia progressiva e incapacitante e eosinofilia sérica, nos líquidos e secreções. A maioria dos pacientes relatava uso prévio de L-triptofano. Sintomas respiratórios são relatados em até $80 \%$ dos casos, eventualmente como manifestação única. 0 tratamento inclui suspensão da droga e corticoterapia. Relatamos o caso de uma mulher de 61 anos com insuficiência respiratória aguda após uso de L-triptofano, hidroxitriptofano e outras drogas. A paciente apresentava eosinofilia no sangue, lavado broncoalveolar e derrame pleural. Após a suspensão da medicação e corticoterapia, houve melhora clínica e radiológica em poucos dias.

Descritores: Insuficiência respiratória; Triptofano; Síndrome de eosinofilia-mialgia.

\begin{abstract}
Eosinophilia-myalgia syndrome was described in 1989 in patients who presented progressive and incapacitating myalgia and eosinophilia in blood, fluids and secretions. Most patients report previous L-tryptophan intake. Respiratory manifestations are found in up to $80 \%$ of the cases, occasionally as the only manifestation. Treatment includes drug discontinuation and administration of corticosteroids. Here, we describe the case of a 61-year-old female admitted with acute respiratory failure after using L-tryptophan, hydroxytryptophan and other drugs. The patient presented eosinophilia, together with elevated eosinophil counts in the bronchoalveolar lavage and pleural effusion. After discontinuation of the drugs previously used, corticosteroids were administered, resulting in clinical and radiological improvement within just a few days.
\end{abstract}

Keywords: Respiratory insufficiency; Tryptophan; Eosinophilia-myalgia syndrome.

\section{Introdução}

Em 1989 foi descrita a síndrome de eosinofilia-mialgia (SEM), que surgiu de forma epidêmica quando diversos pacientes apresentaram mialgia incapacitante e eosinofilia periférica após ingestão de L-triptofano. Isso motivou a retirada do produto do mercado.(1) Desde então, ocorreram mais de 5.000 casos, a maioria em pacientes do sexo feminino e raça branca, média de idade de 49 anos..$^{(1-4)}$

0 L-triptofano é um aminoácido natural encontrado em carnes magras, peixes, leite, queijos, nozes e leguminosas.
É um precursor natural da serotonina (5-HT) e tem sido utilizado para aumentar os níveis orgânicos de 5-HT, para tratamento de depressão, regularização do sono, do apetite sexual, da sensibilidade à dor, da temperatura corporal e melhora das atividades cognitivas. ${ }^{(5)}$

A ingestão de L-triptofano tem sido implicada como agente causal da SEM. ${ }^{(1,6,7)}$ Alguns doentes podem ter queixas respiratórias e alterações radiológicas, e, ocasionalmente, elas aparecem sem envolvimento de outros órgãos.

\footnotetext{
* Trabalho realizado na Disciplina de Pneumologia do Departamento de Clínica Médica da Faculdade de Ciências Médicas - FCM - da Universidade Estadual de Campinas - UNICAMP - Campinas (SP) Brasil.

1. Médico residente da Disciplina de Pneumologia do Departamento de Clínica Médica da Faculdade de Ciências Médicas - FCM - da Universidade Estadual de Campinas - UNICAMP - Campinas (SP) Brasil.

2. Professora Associada da Disciplina de Pneumologia do Departamento de Clínica Médica da Faculdade de Ciências Médicas - FCM - da Universidade Estadual de Campinas - UNICAMP - Campinas (SP) Brasil.

3. Professor Assistente Doutor da Disciplina de Pneumologia do Departamento de Clínica Médica da Faculdade de Ciências Médicas - FCM - da Universidade Estadual de Campinas - UNICAMP - Campinas (SP) Brasil.

4. Professora Doutora da Faculdade de Medicina da Pontifícia Universidade Católica de Campinas - PUC - Campinas (SP) Brasil.

Endereço para correspondência: Tiago de Araujo Guerra Grangeia. Rua das Hortências, 901, Chácara Primavera, CEP 13087-440, Campinas, SP, Brasil.

Tel 5519 3788-7948. Fax 5519 3521-7907. E-mail: tiagoguerra@mpc.com.br

Recebido para publicação em 31/7/2006. Aprovado, após revisão, em 20/12/2006.
} 
Relata-se, a seguir, um caso de insuficiência respiratória aguda presumivelmente relacionada à ingestão de L-triptofano.

\section{Relato de caso}

Mulher de 61 anos, com história de uma semana de dispnéia progressiva, tosse seca e febre. Negava mialgia, exantema, dor torácica, expectoração purulenta, hemoptise, tabagismo, doenças pulmonares prévias ou quaisquer outras doenças crônicas. Referia emagrecimento $(8 \mathrm{~kg}$ ) nos últimos meses e depressão, para o que iniciou três semanas antes do quadro respiratório o uso de uma 'fórmula' que incluía: L-triptofano ( $2 \mathrm{~g} / \mathrm{dia})$, 5-hidroxitriptofano (400 mg/dia), L-taurina, glutamina, inositol, ácido pantotênico, cianocobalamina, piridoxina, L-fosfoserina, fosfotreonina, fluoxetina, carisoprodol e omeprazol.

Ao exame físico, apresentou regular estado geral e febril, sem alterações na ausculta cardíaca. Apresentava-se dispnéica e taquipnéica (freqüência respiratória entre 28 e $40 \mathrm{mrm}$ ). À inspeção e palpação do tórax, observou-se expansibilidade reduzida e simétrica; a ausculta pulmonar revelou crepitações grosseiras e roncos bilateralmente, predominantes no hemitórax esquerdo.

Exames complementares mostraram:

- Gasometria arterial (ar ambiente): $\mathrm{pH}=7,55$; pressão parcial arterial de oxigênio $=53 \mathrm{mmHg}$; pressão parcial de gás carbônico no sangue

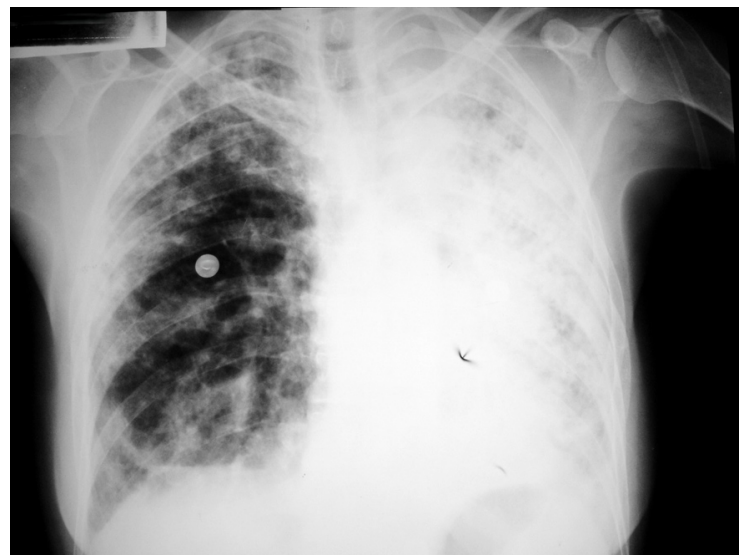

Figura 1 - Radiograma de tórax inicial. Extensas áreas de consolidação, comprometendo todo o pulmão esquerdo e apresentando distribuição predominantemente periférica no pulmão direito. arterial $=23 \mathrm{mmHg}$; saturação periférica de oxigênio $\left(\mathrm{SpO}_{2}\right)=91 \%$;

- Hemograma: 17.180 leucócitos $/ \mathrm{mm}^{3}$, com 9\% de eosinófilos $\left(1.540 / \mathrm{mm}^{3}\right)$;

- Velocidade de hemossedimentação: $120 \mathrm{~mm}$ na primeira hora;

- Eletrólitos, uréia, creatinina, bilirrubinas, enzimas hepáticas, desidrogenase lática (DHL): normais;

- Hemoculturas, urocultura, sorologia para HIV, protoparasitológicos: negativos;

- Radiograma e tomografia computadorizada de tórax iniciais, nas Figuras 1 e 2;

- Ultra-sonografia de tórax: derrame pleural à esquerda, sem septações, que se estendia da base ao terço superior do hemitórax esquerdo;

- Toracocentese: líquido amarelo citrino, com proteína: $3,7 \mathrm{~g} / \mathrm{dL}$ (proteína pleural/ sérica $=0,6), \mathrm{DHL}=242 \mathrm{U} / \mathrm{L}$ (DHL pleural/ sérico $=0,72)$, glicose $=104 \mathrm{mg} / \mathrm{dL}$, 1.960 leucócitos $/ \mathrm{mm}^{3}$ (47,5\% de neutrófilos, $25,5 \%$ de linfócitos, $24,5 \%$ de eosinófilos, 1,5\% de monócitos e 1\% de basófilos) e 3.000 hemácias $/ \mathrm{mm}^{3}$. Pesquisas e culturas para fungos e bactérias: negativas;

- Broncoscopia: lavado broncoalveolar (LBA) com 53.000 células $/ \mathrm{mL}$, com pesquisas e culturas para fungos e micobactérias negativas, e cultura positiva para Staphylococcus aureus;

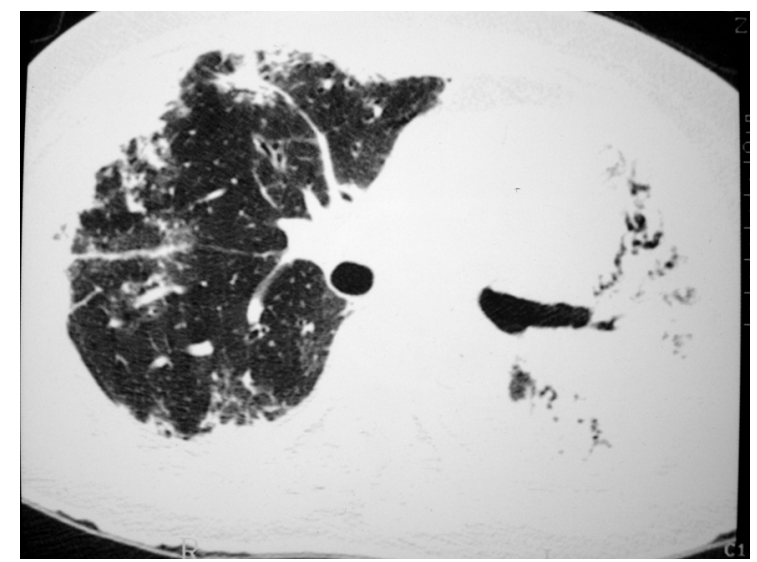

Figura 2 - Tomografia computadorizada de tórax inicial. Áreas de consolidação difusas no pulmão esquerdo, com desvio da linha de junção anterior para a esquerda (perda volumétrica) e broncogramas aéreos; consolidações periféricas e áreas de vidro fosco no pulmão direito. 
- Citologia (LBA): exsudato inflamatório crônico ativo purulento com freqüentes eosinófilos, siderófagos e células gigantes multinucleadas do tipo corpo estranho; e

- Biópsia transbrônquica: foco de reação granulomatosa incipiente não necrosante em região broncovascular, com culturas negativas.

A paciente foi tratada inicialmente para pneumonia comunitária grave com antibioticoterapia (ceftriaxone e levofloxacin) e oxigenoterapia. Após uma semana mantinha febre, hipoxemia e dispnéia aos mínimos esforços, sem melhora radiológica. Houve aumento da eosinofilia sérica $\left(2.670 / \mathrm{mm}^{3}\right.$ $16 \%$ ), que associada à eosinofilia pleural e no LBA, motivaram tratamento com metilprednisolona na dose de $125 \mathrm{mg}$ a cada $6 \mathrm{~h}$.

A evolução após a introdução da corticoterapia foi brilhante: houve resolução da febre e da eosinofilia em três dias; após uma semana, melhora da hipoxemia $\left(\mathrm{SpO}_{2}=94 \%\right.$ em ar ambiente) e das alterações radiológicas, restando apenas imagem cística no pulmão esquerdo que não foi mais detectada em radiografias posteriores, sendo assim interpretada como pneumatocele (Figura 3).

Após 15 dias a paciente não apresentava queixas e mantinha $\mathrm{SpO}_{2}=96 \% \mathrm{em}$ ar ambiente. Recebeu alta hospitalar com $60 \mathrm{mg}$ de prednisona, reduzida gradativamente até a suspensão em quatro meses. Atualmente apresenta fraqueza em musculatura proximal de membros inferiores, sem queixas respiratórias.
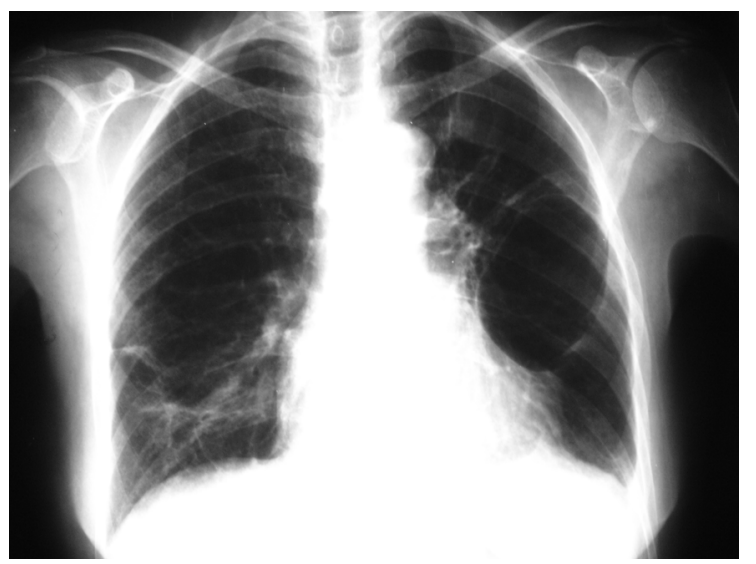

Figura 3 - Radiograma de tórax após uma semana de tratamento. Não se observam mais em radiogramas de tórax posteriores: as áreas de consolidação; opacidades heterogêneas em base pulmonar direita, lineares; ou imagem cística em campo médio de hemitórax esquerdo (pneumatocele).

\section{Discussão}

A paciente apresentava insuficiência respiratória aguda associada a consolidações pulmonares bilaterais, aumento do número de eosinófilos no sangue, no LBA e no derrame pleural. Nos diagnósticos diferenciais de eosinofilia pulmonar foram considerados pouco prováveis: a pneumonia eosinofílica crônica, pela apresentação clínica aguda; síndrome de Churg Strauss, pois não havia sinais de neuropatia periférica ou antecedente de asma; eosinofilia pulmonar simples, devido ao exame protoparasitológico negativo e à apresentação clínica grave; aspergilose broncopulmonar alérgica, dada a ausência de história de asma, bronquiectasias ou fibrose cística; ou síndrome hipereosinofílica, pela ausência de comprometimento extrapulmonar.

Havia clara relação temporal entre o início do quadro clínico e o uso das diversas substâncias, tornando mais provável a hipótese de eosinofilia pulmonar induzida por drogas. Dentre as substâncias utilizadas, o L-triptofano e o hidroxitriptofano podem estar associados à presença de infiltrados pulmonares e eosinofilia. ${ }^{(1,8)}$ A fluoxetina pode estar associada à pneumonite intersticial subaguda, porém, não há relato de eosinofilia pulmonar. ${ }^{(9)}$

Por ocasião da descrição da SEM, os critérios diagnósticos incluíam mialgia incapacitante, eosinofilia periférica $>1.000 / \mathrm{mm}^{3}$ e ausência de infecção ou neoplasia que justificasse o quadro

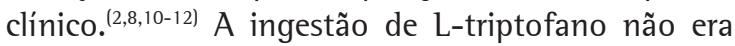
um critério diagnóstico; no entanto, 86 a 97\% dos pacientes a referiam, com doses que variavam entre 500 a $12.000 \mathrm{mg} / \mathrm{dia}$, e intervalo entre o início da ingestão e o surgimento do quadro clínico de quatro semanas a seis anos. ${ }^{(2-4)} \mathrm{A}$ associação descrita sugeria nexo causal entre a ingestão da droga e a SEM, embora tal relação não pudesse ser totalmente comprovada. ${ }^{(6,7,13)}$ Autores consideram que o acúmulo de metabólitos de L-triptofano seja o responsável pelas manifestações clínicas. ${ }^{(1)}$

Os inúmeros casos de SEM descritos ampliaram a gama de sintomas e sinais clínicos, permitindo definir que a doença se caracteriza por uma fase aguda, com duração aproximada de quatro meses, em que podem ocorrer mialgia incapacitante, febre, emagrecimento, fraqueza, dispnéia, tosse, dor torácica, edema periférico, artralgias, exantema, parestesias, hiperestesias, espessamento cutâneo, prurido, dispepsia e palpitações, entre outros..$^{(2,8,11,14)}$ 
$\mathrm{Na}$ fase crônica, predominam fadiga e sintomas neurológicos (déficits de atenção e memória). ${ }^{(12)}$

Sintomas respiratórios são comuns na SEM e podem estar presentes em até $80 \%$ dos pacientes, ${ }^{(14)}$ inclusive como manifestação única. Em uma série de 1.531 casos, ${ }^{(15)}$ tosse ou dispnéia estiveram presentes em 611 deles (59\%), e em outro estudo, ${ }^{(16)}$ com 118 pacientes, foram encontrados sintomas respiratórios em 64\%, alterações radiológicas em 16\% e derrames pleurais em 15\%. 0 acometimento pulmonar confere pior prognóstico e alguns pacientes podem evoluir para insuficiência respiratória aguda. ${ }^{(3,4,17)}$

Os exames complementares, em geral, evidenciam leucocitose e eosinofilia (valores superiores a $3.000 / \mathrm{mm}^{3}$ são mais sugestivos). ${ }^{(11)} 0$ radiograma de tórax pode ser normal, apresentar opacidades intersticiais lineares ou reticulonodulares, consolidações pulmonares difusas e derrame pleural. ${ }^{(3,4,10)}$ Os derrames pleurais podem aparecer em até um terço dos casos, e geralmente são exsudatos eosinofílicos. ${ }^{(4,10,16)}$

De um total de 12 casos relatados, cinco foram submetidos à tomografia de tórax. ${ }^{(4,17,18)}$ Em quatro pacientes foram detectadas consolidações, em geral focais e de predomínio basal, em um paciente foi detectado derrame pleural e em um paciente foram descritos apenas nódulos peribronquiolares difusos. Não houve relatos de consolidações extensas. 0 pequeno número de pacientes não nos permite estabelecer um padrão, embora consolidações tenham sido encontradas em 80\% dos pacientes.

A espirometria pode ser normal ou evidenciar distúrbio ventilatório obstrutivo ou restritivo. ${ }^{(3,10,14)}$

A síndrome é definida com base em critérios clínicos e radiológicos, não sendo obrigatória a presença de biópsia compatível, mesmo porque não há um padrão típico. Diversas são as alterações histopatológicas descritas: pneumonite intersticial com predomínio de linfócitos, vasculite transmural e infiltrado inflamatório perivascular, ${ }^{(14)}$ vasculite granulomatosa, ${ }^{(2,3)}$ pleurite fibrinosa e bronquiolite folicular. ${ }^{(3)}$ No caso relatado, o fragmento da biópsia transbrônquica mostrou foco de reação granulomatosa incipiente não necrosante em região broncovascular, achados compatíveis com uma vasculite granulomatosa semelhante ao caso descrito em um estudo. ${ }^{(3)} \mathrm{A}$ biópsia a céu aberto, que permitiria uma análise mais completa dos achados histopatológicos, não foi realizada, pois o quadro de falência respiratória aguda nos fez optar pela instituição de tratamento imediato, e a resposta rápida ao mesmo desobrigou-nos de realizar o procedimento.

0 tratamento da SEM consiste na suspensão da droga e no uso de costicosteróides: 5 a $60 \mathrm{mg}$ de prednisona ao dia por quatro semanas, com posterior redução gradual da dose. Cerca de $80 \%$ dos pacientes apresentam melhora dos sintomas, a maioria já nos primeiros três dias de tratamento, o que foi observado com esta paciente. Os sintomas mais refratários são fadiga e sintomas neurológicos (cognitivos). A mortalidade estimada é de 2 a $6 \%$, sendo a maioria dos óbitos devida à polineuropatia ascendente com insuficiência respiratória. ${ }^{(1)}$

Pelo quadro clínico de insuficiência respiratória aguda associado à eosinofilia periférica e pulmonar, em um contexto no qual outras doenças eosinofílicas seriam menos prováveis, é possível suspeitar de um nexo causal entre a doença aguda apresentada e a ingestão de L-triptofano.

0 L-triptofano é uma substância largamente utilizada no nosso meio, de maneira indiscriminada e sem controle de receituário médico, pois faz parte de diversas 'fórmulas' empregadas em tratamentos ditos 'alternativos', como suplementos nutricionais ou na 'medicina ortomolecular'. Assim, é importante que estejamos atentos à possibilidade do surgimento deste vasto espectro de manifestações clínicas, que em um extremo de gravidade pode evoluir com insuficiência respiratória aguda e morte.

\section{Referências}

1. Ahmed MM, Mubashir E, Sairam S, Lisse JR. EosinophiliaMyalgia Syndrome. [monograph on the Internet]. e-medicine; WebMD; c1996-2006 [cited 2006 May 5]. Available from: http://www.emedicine.com/med/topic693. htm\#section workup

2. Herrick MK, Chang Y, Horoupian DS, Lombard CM, Adornato BT. L-tryptophan and the eosinophilia-myalgia syndrome: pathologic findings in eight patients. Hum Pathol. 1991;22(1):12-21.

3. Tazelaar HD, Myers JL, Drage CW, King TE Jr, Aguayo S, Colby TV. Pulmonary disease associated with L-tryptophaninduced eosinophilic myalgia syndrome. Clinical and pathologic features. Chest. 1990;97(5):1032-6.

4. Campagna AC, Blanc PD, Criswell LA, Clarke D, Sack KE, Gold WM, Golden JA. Pulmonary manifestations of the eosinophilia-myalgia syndrome associated with tryptophan ingestion. Chest. 1992;101(5):1274-81.

5. Ballone GJ. Serotonina. [monograph on the Internet] Psiqweb; c2005 [cited 2006 Oct 4]. Available from: http:// virtualpsy.locaweb.com.br/index.php?sec=61 ctart=295

6. Shapiro S. L-tryptophan and eosinophilia-myalgia syndrome. Lancet. 1994;344(8925):817-9. 
7. Shapiro S. Epidemiologic studies of the association of L-tryptophan with the eosinophilia-myalgia syndrome: a critique. J Rheumatol Suppl. 1996;46:44-58; discussion 58-9.

8. Clauw DJ, Pincus T. The eosinophilia-myalgia syndrome: what we know, what we think we know, and what we need to know. J Rheumatol Suppl. 1996;46:2-6.

9. de Kerviler E, Trédaniel J, Revlon G, Groussard 0, Zalcman G, Ortoli JM, et al. Fluoxetin-induced pulmonary granulomatosis. Eur Respir J. 1996;9(3):615-7.

10. Williamson MR, Edison M, Rosenberg RD, Williamson SL. Eosinophilia-myalgia syndrome: findings on chest radiographs in 18 patients. Radiology. 1991;180(3):849-851.

11. Hertzman PA. Criteria for the definition of the eosinophiliamyalgia syndrome. J Rheumatol Suppl. 1996;46:7-12.

12. Pincus T. Eosinophilia-myalgia syndrome: patient status 2-4 years after onset. J Rheumatol Suppl. 1996;46:19-24; discussion 24-5.
13. Margolin L. Non-L-tryptophan related eosinophilia-myalgia syndrome with hypoproteinemia and hypoalbuminemia. J Rheumatol. 2003;30(3):628-9.

14. Silver RM. Pathophysiology of the eosinophilia-myalgia syndrome. J Rheumatol Suppl. 1996;46:26-36.

15. Swygert LA, Maes EF, Sewell LE, Miller L, Falk H, Kilbourne EM. Eosinophilia-myalgia syndrome. Results of national surveillance. JAMA. 1990;264(13):1698-703.

16. Centers for Disease Control (CDC). Clinical spectrum of eosinophilia-myalgia syndrome--California. MMWR Morb Mortal Wkly Rep. 1990;39(6):89-91.

17. Banner AS, Borochovitz D. Acute respiratory failure caused by pulmonary vasculitis after L-tryptophan ingestion. Am Rev Respir Dis. 1991;143(3):661-4.

18. Strumpf 1J, Drucker RD, Anders KH, Cohen S, Fajolu 0. Acute eosinophilic pulmonary disease associated with the ingestion of L-tryptophan-containing products. Chest. 1991;99(1):8-13. 